\title{
A Conservative Surgical Approach to the Treatment of Optic Disc Pit Maculopathy: Four Case Reports
}

\author{
Emilio Rapizzi ${ }^{a}$ Paola Gallon ${ }^{b}$ Diego Ponzin ${ }^{b}$ Stefano Ferrari ${ }^{b}$ \\ Nicola Zemella ${ }^{a}$ \\ aDepartment of Ophthalmology, Ospedale dell'Angelo, ULSS3 Serenissima, Venice, Italy; \\ ${ }^{b}$ Fondazione Banca degli Occhi del Veneto, Venice, Italy
}

\section{Keywords}

Optic disc pit maculopathy - Vitrectomy - Laser - Gas tamponade - Posterior vitreous detachment

\begin{abstract}
The purpose of this report is to present the outcomes of surgical interventions in 4 patients with maculopathy associated with optic disc pit (ODP). We report 4 cases of patients affected by ODP maculopathy and treated by core vitrectomy with induction of posterior vitreous detachment and peeling of the internal limiting membrane restricted to the interpapillary macular zone without laser treatment and gas tamponade. The patients had rapid resolution of the multilayer inner retinoschisis-like separation and progressive slow reabsorption of the macular intraretinal and subretinal fluid up to complete retinal reattachment. Currently, there are still no widely accepted guidelines related to the best technique in the management of the maculopathy associated with ODP. We used a conservative approach, without the adoption of intravitreal gas injection or laser.


 Ophthalmology}

\section{Introduction}

Optic disc pit (ODP) is a rare congenital anomaly usually characterized by a single, grayish, oval-shaped depression at the optic disc level. Its incidence is around 1 in 11,000, unilateral in $85 \%$ of cases, without a prevalence between males and females [1]. In about twothirds of patients the malformation is complicated by the development of ODP maculopathy (ODP-M), with accumulation of intraretinal fluid, subretinal fluid, and retinal pigment changes. ODP-M is the cause of serious visual defects and normally arises between 20 and 40 years of age [2]. The exact origin of this liquid is still unknown. To date, there are four proposed theories about its origin: (1) vitreous fluid that enters the subretinal space through the optic pit, (2) cerebrospinal fluid which enters into the intra- and subretinal spaces from the subarachnoid space through the ODP defects, (3) fluid leakage from blood vessels at the ODP (but there are no angiographic findings), or (4) fluid from the choroid through Bruch's membrane and peripapillary atrophy (unlikely) $[1,3]$. In any case, the accumulation of fluid is a very debilitating complication.

A problem associated with ODP-M is that there are no clear guidelines for clinical and surgical management. About $25 \%$ of cases have spontaneous regression of the macular detachment [4]. In the other cases, various therapeutic approaches have been proposed, but none has been established as the treatment of choice. Laser photocoagulation at the temporal disc margin could be a possibility. It creates a chorioretinal scar that acts as a barrier to fluid migration. However, laser treatment could itself be the source of significant visual field defects. Another treatment currently accepted for ODP-M is intravitreal gas injection, as pneumatic displacement improves visual acuity (VA) by reattachment of the macula [1, 3]. However, only a few reports have been published, with reattachment achieved in approximately half of the cases and sometimes with only a temporary effect due to recurrence [5-7]. Moreover, the use of gas could lead to cataract, a very undesirable effect considering the young age of the patients who normally have ODP-M. An alternative approach is macular buckling surgery, but it is a complicated surgical procedure that needs an implant to be fixed to the posterior part of the eye globe. Because of such hurdles, the technique has not gained much popularity, albeit its promising results [8]. Even if there is not effective proof, vitreous traction is probably one of the key elements in the pathogenesis of ODP-M. For this reason, currently, in case of ODP-M, the most used technique is pars plana vitrectomy (PPV). In most cases, PPV is associated with gas tamponade. However, gas tamponade is not the best choice since ODP-M is a disease that normally affects patients between the second and third decades of life.

Hirakata et al. [9] reported that vitrectomy with induction of posterior vitreous detachment (PVD) and gas tamponade, without additional laser treatment, is an effective method to reattach the macula and improve VA. The same group evaluated the clinical outcomes of 8 consecutive eyes undergoing vitrectomy with PVD induction without performing gas tamponade or laser application [10].

With the hypothesis that gas tamponade may not be necessary for the treatment of ODP-M and that the best surgery should be the least invasive possible, we report 4 patients treated with a "conservative" surgical approach. In all of these cases we performed vitrectomy ( 25 or $27 \mathrm{G}$ ) with induction of PVD and peeling of the internal limiting membrane (ILM) restricted to the interpapillary macular zone. This technique allowed to reduce all anomalous vitreous-papillary tractions without the use of laser or gas, therefore sparing the lens. We present the results of these cases and describe the course of retinal reattachment after surgery for ODP-M. 


\section{Case Report 1}

The first case was a young man aged 24 years with ODP-M. He had never received previous treatments. After being monitored for 3 months with no spontaneous resolution, vitrectomy with induction of PVD and partial peeling of the ILM was performed. Before the surgery, he had a VA of 20/70. At the first postsurgical visit, anatomical positive results started to appear, with resolution of the multilayer inner retinoschisis-like separation. At the 3-month follow-up, fluid accumulation was still very evident although less than previously. At the 7th month, VA was 20/40 and the presence of intra- and subretinal fluid was found to be significantly reduced, as detected by optical coherence tomography as well as infrared and fundus autofluorescence photographs. Excellent results were achieved starting from the 13th month, with a VA of 20/20, complete retinal reattachment after optical coherence tomography analysis, and diffused faint granular hyperfluorescence after autofluorescence detection (Fig. 1).

\section{Case Report 2}

The second case was a 35-year-old man with ODP-M with no previous treatments. Similarly to case 1, he was monitored for 3 months to see whether spontaneous fluid reabsorption would occur and then treated with the same surgical technique. Also in this case, macular detachment decreased gradually with almost complete resolution after 8 months. Both functional and anatomical improvements were already visible 10 days after surgery. VA was 20/70 before surgery and 20/25 8 months after surgery (Fig. 2).

\section{Case Report 3}

The third case was a 39-year-old man suffering from ODP-M since 1 year. Because of the worsening of his VA (from 20/25 to 20/40 within 4 months), a surgical intervention was scheduled. The patient's initial clinical situation was less compromised than in the first two cases, with complete resolution of the pathology 6 months after surgery. In this period, VA went from 20/40 to 20/25 and the amount of intraretinal fluid at the macular level decreased considerably (Fig. 3).

\section{Case Report 4}

The last case, a 67-year-old man, underwent a surgical intervention because of deterioration of the ODP-M from which he had suffered for more than 4 years. As before, at the beginning there was an anatomical improvement, with functional improvements appearing later. During the 9 months of follow-up observations, VA improved from 20/100 (presurgery) to $20 / 50$ (at 7 months) and finally to $20 / 40$ (at 9 months). Improvements were lower than what we observed for the previous cases, thus suggesting a potential role of the duration of ODP-M in postoperative results. Preoperative condition of ODP-M, progression over time (up to 9 months after surgery), and the progressive reduction in the accumulated fluid are shown in Figure 4. 


\section{Discussion}

Among all theories explaining the pathogenesis of ODP-M [1, 3], we believe that the most accredited is that there is a vitreous traction around the ODP causing passive fluid migration into the intra- and subretinal spaces. To treat our patients affected by ODP-M, we followed the technique described Hirakata et al. [10]. The same group previously used a similar technique, with the main difference that gas tamponade was also used [9]. We believe that the use of gas should be avoided because it has been reported to increase intraocular pressure and to induce abnormal vitreoretinal tractions, intraocular inflammation, and cataract. Thus, based on the functional and anatomical results obtained by Hirakata et al. [10], we performed lens-sparing core vitrectomy ( 25 or $27 \mathrm{G}$ ), with induction of PVD and peeling of the ILM restricted to the interpapillary macular zone. Our aim was to reduce the anomalous vitreous traction without the necessity of using laser or gas. All surgeries were performed by the same physician between 2017 and 2018. During the surgical procedures, no intra- or postoperative complications were reported.

The four patients had a similar clinical course, with both functional and anatomical improvements being observed. In all cases we observed rapid resolution of the multilayer inner retinoschisis-like separation and progressive slow reabsorption of the macular intra- and subretinal fluid up to complete retinal reattachment as well as progressive VA improvement. As previously reported $[9,10]$, the slow reabsorption of subretinal fluid that we observed is probably due to limited access to the subretinal space, fluid composition, and retinal pigment epithelium cell metabolic changes. The main advantages of our technique are that (1) it is a "conservative" procedure, (2) it reduces the risk of cataract progression/formation and other intraocular complications due to gas tamponade, and (3) it eliminates the need to place the patient in a prone postoperative position, with faster postsurgical recovery.

However, there are other theories. There is some evidence that PPV alleviates only vitreous traction and can lead to improvements only in cases where the pathogenesis of ODP-M is related to the vitreous. Macular buckling has been reported as an alternative strategy for the treatment of ODP-M and seems to provide favorable results, independent of the origin of fluid. Theodossiadis and colleagues $[8,11,12]$ first used this technique and reported a success rate of approximately $85 \%$ regarding fluid absorption after a long mean follow-up of 13 years. The rationale behind this technique is that in cases where the fluid comes from the vitreous cavity, the macular buckling ends up obstructing the entrance of fluid into the intraretinal space, thus alleviating the vitreous traction. In cases where the fluid originates from the subarachnoid space, it does not permit fluid circulation towards the subretinal space. However, the technique is difficult and has a long learning curve, which explains why it is not widely applied and only limited to the experience of a few surgeons.

In conclusion, despite its small size, our case report of 4 patients is in line with all the major published works, which have so far reported series of limited patient numbers (ranging between 3 and 18 patients) [1]. In addition, our results mirror those reported in the literature: for example, in case 1, we had complete resolution within 13 months, comparable with the average of 12 months reported by Hirakata et al. [10].

The clinical evidence reported in this paper is leading us to continue in this direction, i.e., treating patients at the beginning of the ODP-M manifestations (after observation of no spontaneous resolution) and performing a conservative surgical technique, without the use of gas tamponade or laser treatment. 


\section{Acknowledgments}

The authors would like to thank the nurses and the whole staff of the Department of Ophthalmology for assistance and help.

\section{Statement of Ethics}

This research was conducted ethically in accordance with the World Medical Association Declaration of Helsinki. The subjects provided written informed consent for their cases to be published, including publication of images.

\section{Disclosure Statement}

The authors have no financial or conflicting interests to disclose.

\section{Funding Sources}

No financial support or research funding was received for this study.

\section{Author Contributions}

E. Rapizzi and N. Zemella treated the patients, including surgeries and follow-up visits. P. Gallon and D. Ponzin collected the data, analyzed the findings, and worked on the manuscript. S. Ferrari wrote and revised the manuscript. All authors read and approved the final version of the manuscript.

\section{References}

1 Moisseiev E, Moisseiev J, Loewenstein A. Optic disc pit maculopathy: when and how to treat? A review of the pathogenesis and treatment options. Int J Retina Vitreous. 2015 Aug;1(1):13.

2 Postel EA, Pulido JS, McNamara JA, Johnson MW. The etiology and treatment of macular detachment associated with optic nerve pits and related anomalies. Trans Am Ophthalmol Soc. 1998;96:73-88.

3 Uzel MM, Karacorlu M. Optic disk pits and optic disk pit maculopathy: a review. Surv Ophthalmol. 2019 Sep-0ct;64(5):595-607.

4 Sugar HS. Congenital pits in the optic disc and their equivalents (congenital colobomas and colobomalike excavations) associated with submacular fluid. Am J Ophthalmol. 1967 Feb;63(2):298-307.

5 Lincoff $\mathrm{H}$, Kreissig I. Optical coherence tomography of pneumatic displacement of optic disc pit maculopathy. Br J Ophthalmol. 1998 Apr;82(4):367-72.

6 Akiyama H, Shimoda Y, Fukuchi M, Kashima T, Mayuzumi H, Shinohara Y, et al. Intravitreal gas injection without vitrectomy for macular detachment associated with an optic disk pit. Retina. 2014 Feb;34(2):222-7.

7 Lei L, Li T, Ding X, Ma W, Zhu X, Atik A, et al. Gas tamponade combined with laser photocoagulation therapy for congenital optic disc pit maculopathy. Eye (Lond). 2015 Jan;29(1):106-14.

8 Theodossiadis GP, Theodossiadis PG. The macular buckling technique in the treatment of optic disk pit maculopathy. Semin Ophthalmol. 2000 Jun;15(2):108-15.

9 Hirakata A, Okada AA, Hida T. Long-term results of vitrectomy without laser treatment for macular detachment associated with an optic disc pit. Ophthalmology. 2005 Aug;112(8):1430-5.

10 Hirakata A, Inoue M, Hiraoka T, McCuen BW 2nd. Vitrectomy without laser treatment or gas tamponade for macular detachment associated with an optic disc pit. Ophthalmology. 2012 Apr;119(4):810-8. 
Rapizzi et al:: A Conservative Surgical Approach to the Treatment of Optic Disc Pit Maculopathy

11 Theodossiadis GP, Chatziralli IP, Theodossiadis PG. Macular buckling in optic disc pit maculopathy in association with the origin of macular elevation: 13-year mean postoperative results. Eur J Ophthalmol. 2015 May-Jun;25(3):241-8.

12 Chatziralli I, Theodossiadis P, Theodossiadis GP. Optic disk pit maculopathy: current management strategies. Clin Ophthalmol. 2018 Aug;12:1417-22. 


\section{Case Reports in Ophthalmology}

Case Rep Ophthalmol 2020;11:196-204

DOI: $10.1159 / 000507880$

(c) 2020 The Author(s). Published by S. Karger AG, Basel www.karger.com/cop

Rapizzi et al.: A Conservative Surgical Approach to the Treatment of Optic Disc Pit Maculopathy
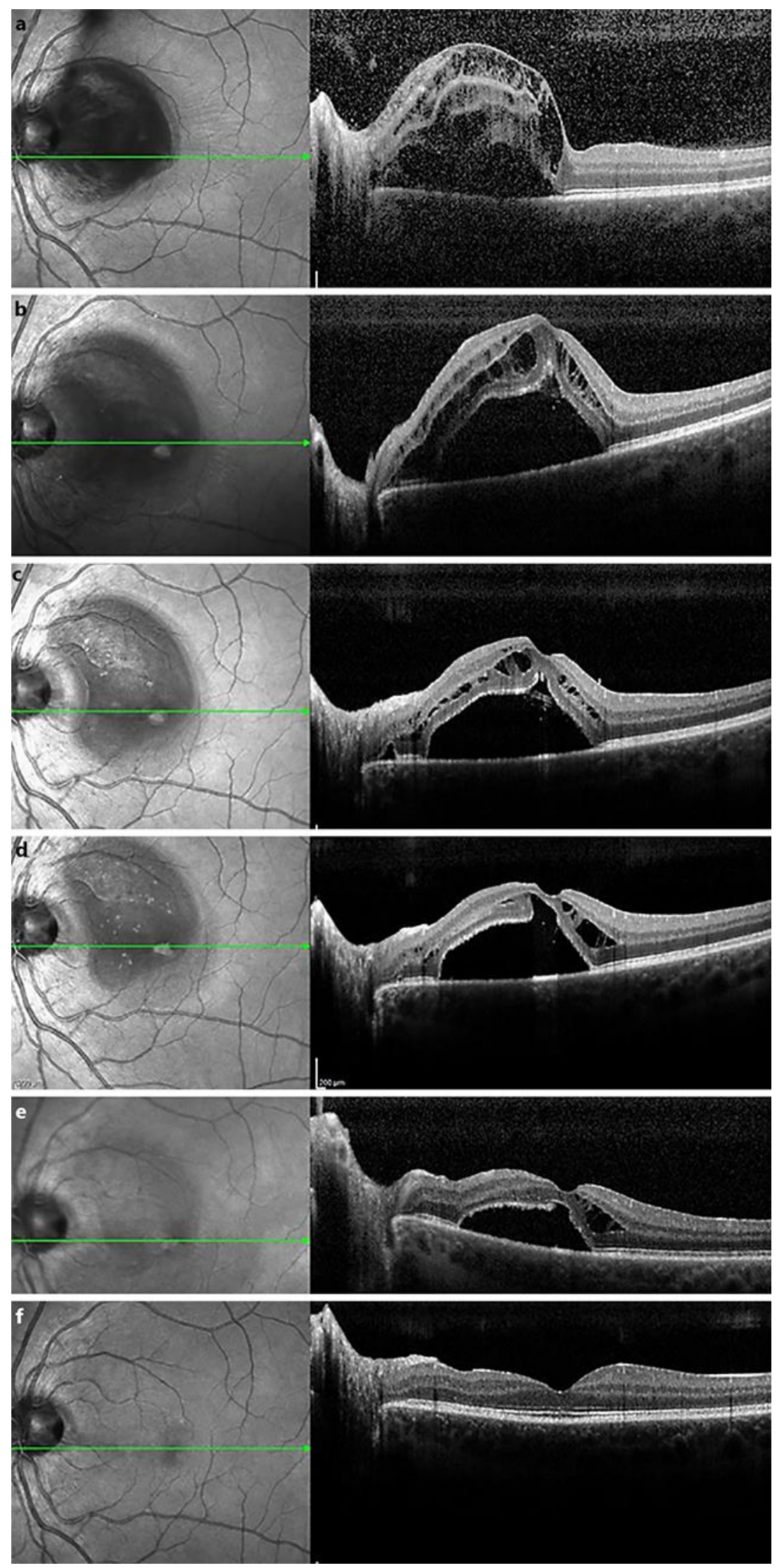


\section{Case Reports in Ophthalmology}

\begin{tabular}{l|l}
\hline Case Rep Ophthalmol 2020;11:196-204 \\
\hline DOI: 10.1159/000507880 & $\begin{array}{l}\text { @ 2020 The Author(s). Published by S. Karger AG, Basel } \\
\text { www.karger.com/cop }\end{array}$ \\
\hline
\end{tabular}

Rapizzi et al.: A Conservative Surgical Approach to the Treatment of Optic Disc Pit Maculopathy

Fig. 1. Representative clinical findings from patient 1. a Presurgery. b Two days after surgery. c Seven days after surgery. $\mathbf{d}$ Three months after surgery. e Seven months after surgery. $\mathbf{f}$ Thirteen months after surgery.
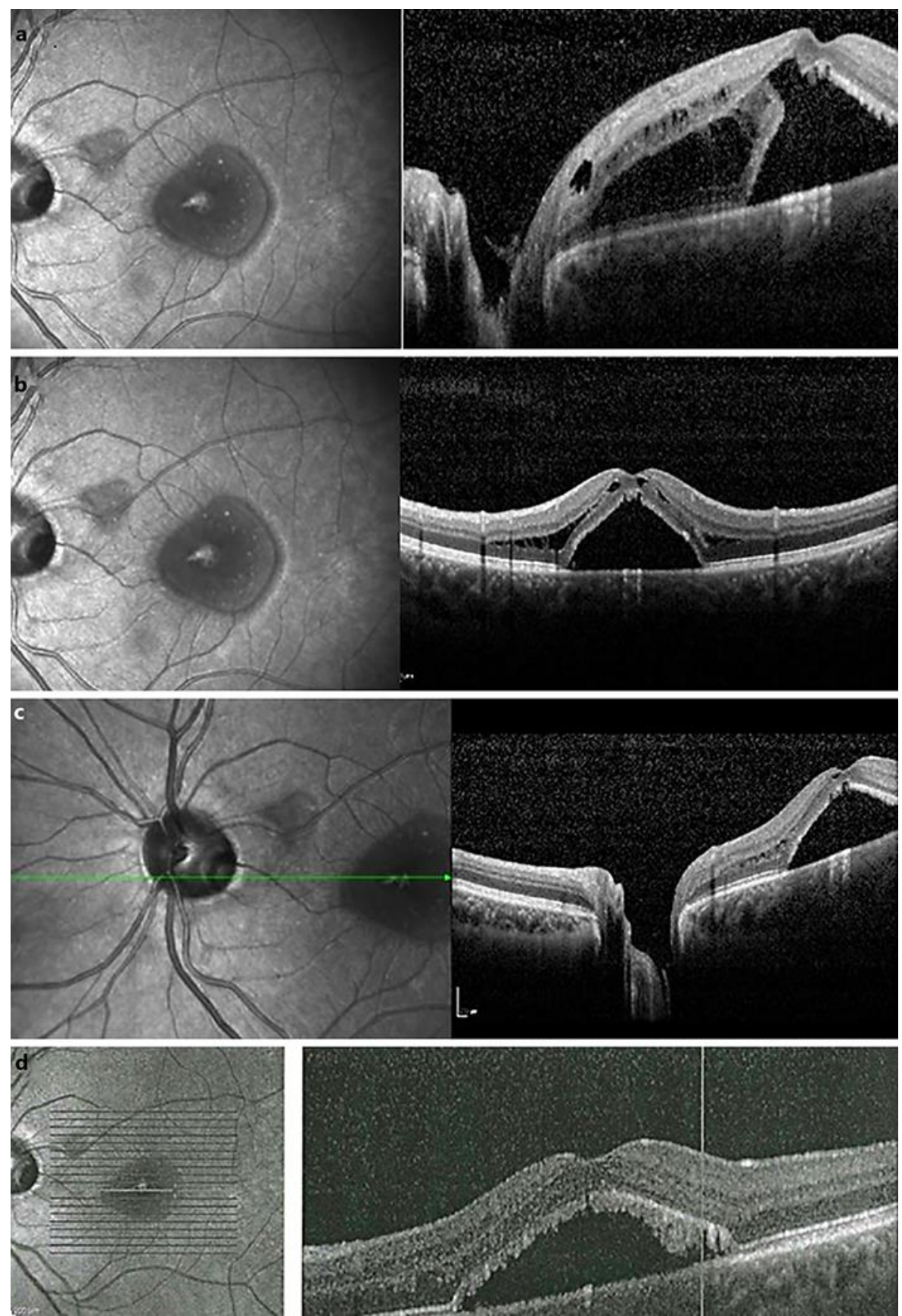

Fig. 2. Representative clinical findings from patient 2. a Presurgery. b Ten days after surgery. $\mathbf{c}$ Two months after surgery. $\mathbf{d}$ Eight months after surgery. 


\section{Case Reports in Ophthalmology}

www.karger.com/cop
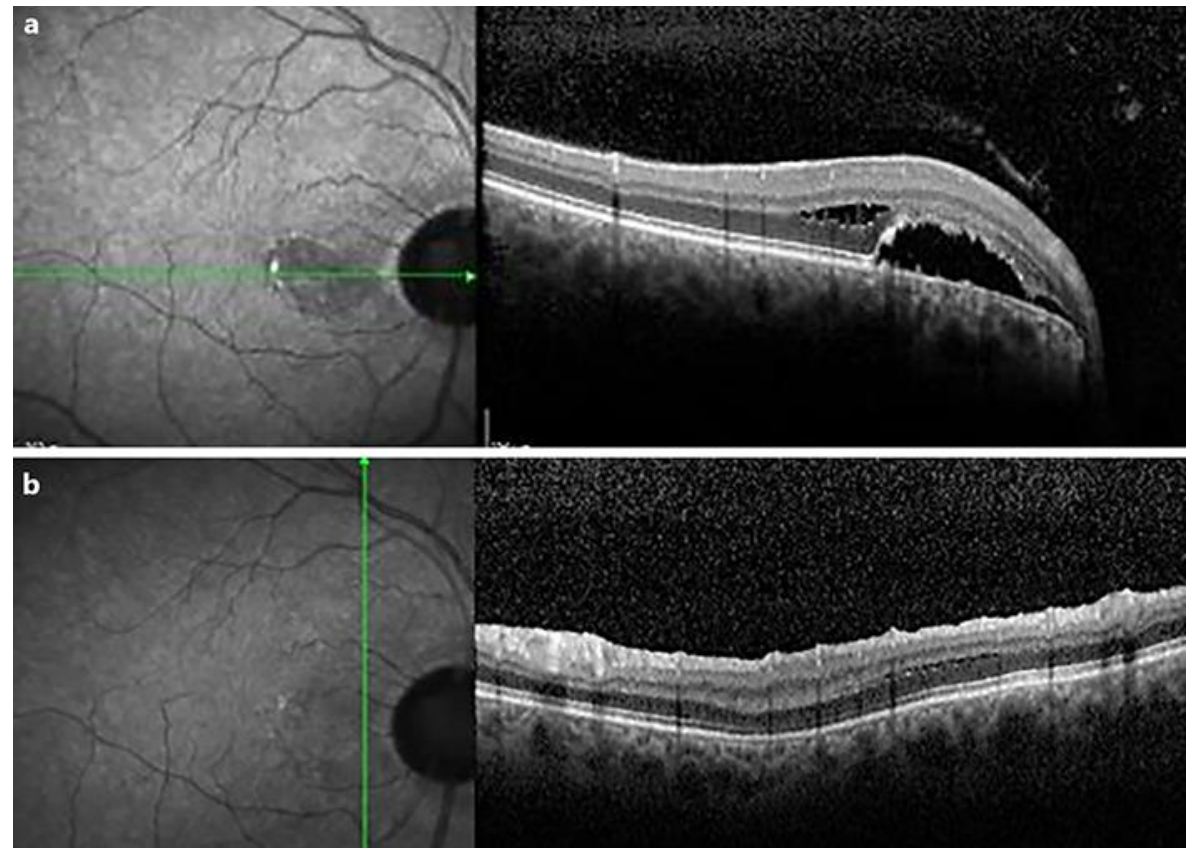

Fig. 3. Representative clinical findings from patient 3. a Presurgery. b Six months after surgery.
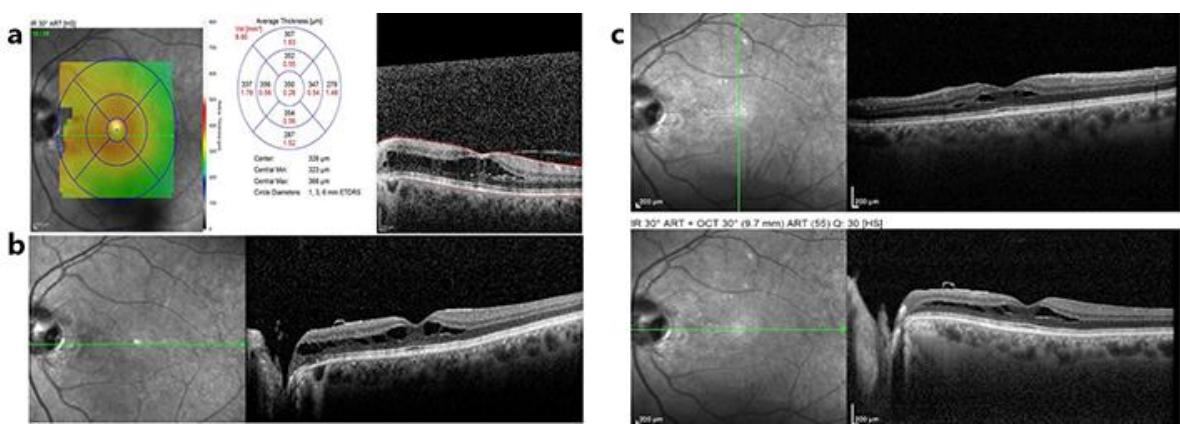

Fig. 4. Representative clinical findings from patient 4. a Presurgery. b Four months after surgery. c Nine months after surgery. 